\title{
DUMBELL - A PROGRAM TO CALCULATE THE STRUCTURE AND THERMODYNAMICS OF A CLASSICAL FLUID OF HARD, HOMONUCLEAR DIATOMIC MOLECULES
}

\author{
F. LADO \\ Department of Physics, North Carolina State University, Raleigh, NC 27695-8202, USA
}

Received 22 March 1985

\section{PROGRAM SUMMARY}

Title of program: DUMBELL

Catalogue number: AADS

Program obtainable from: CPC Program Library, Queen's University of Belfast, N. Ireland (see application form in this issue)

Computer: IBM 3081; Installation: Triangle Universities Computation Center

Operating system: MVS

Programming language used: FORTRAN 77

High speed storage required: 140 Kwords

Number of bits in a word: 32

Peripherals used: terminal, printer

Number of lines in combined program and test deck: 983

Keywords: molecular fluids, Percus-Yevick analytic model, Ornstein-Zernike equation

Nature of the physical problem

Calculation of the spherical harmonic coefficients of the pair distribution, direct correlation and other functions of a fluid of hard, homonuclear diatomic molecules and from these its thermodynamics.

\section{Method of solution}

Spherical harmonic coefficients of the molecular direct correlation function $C(12)$ are obtained from an ad hoc but surprisingly effective generalization of the Percus-Yevick hard sphere solution [1,2]. These are Fourier transformed to yield, through the Ornstein-Zernike equation, the transform of the series function $S(12)$. After inverse Fourier transformation of these, the function $S(12)$ is reconstructed from its coefficients and used finally to generate the spherical harmonic coefficients of the pair distribution function $g(12)$ in Percus-Yevick approximation.

Restrictions on the complexity of the problem

Up to 14 coefficients can be used in the spherical harmonic expansions.

Typical running time

The test job, using five harmonic coefficients, takes $81 \mathrm{~s}$ on an IBM 3081; with 14 coefficients, the running time is $130 \mathrm{~s}$.

\section{References}

[1] R. Pynn, J. Chem. Phys. 60 (1974) 4579.

[2] F. Lado, Mol. Phys. 54 (1985) 407. 


\section{LONG WRITE-UP}

\section{Introduction}

The Ornstein-Zernike $(\mathrm{OZ})$ equation for a fluid of linear molecules at density $\rho[1]$.

$$
g(12)-1=C(12)-\frac{\rho}{4 \pi} \int \mathrm{d} 3 C(13)[g(32)-1],
$$

couples the pair distribution function (PDF) $g(12)$ and the direct correlation function (DCF) $C(12)$, so that given $C(12), g(12)$ and thereby the thermodynamics of the fluid can be determined. For a pair of molecules labelled 1 and 2. these functions depend both on the separation $\boldsymbol{r}_{12}$ between molecular centers and on the orientations $\omega_{1}=\left(\theta_{1}, \phi_{1}\right)$ and $\omega_{2}=\left(\theta_{2}, \phi_{2}\right)$ of the molecular axes with respect to some roordinate frame; i.e.,

$g(12)=g\left(\boldsymbol{r}_{12}, \boldsymbol{\theta}_{1}, \phi_{1}, \boldsymbol{\theta}_{2}, \phi_{2}\right)$

and similarly for $C(12)$, while in eq. (1) we have put

$\int \mathrm{d} 3=\int \mathrm{d} \boldsymbol{r}_{3} \mathrm{~d} \omega_{3}=\int \mathrm{d} \boldsymbol{r}_{3} \mathrm{~d} \phi_{3} \mathrm{~d} \theta_{3} \sin \theta_{3}$.

For simple fluids with spherically symmetric potentials, the solution of eq. (1) usually proceeds through a deconvoluting Fourier transform. This route can also be followed for molecular fluids. albeit with more travail [2], and forms the substance of the program DUMBELL.

Since $g(12)$ is discontinuous for hard potentials, it is numerically advantageous to first calculate instead the continuous function

$S(12)=g(12)-1-C(12)$

from eq. (1) and then determine $g(12)$ using the closure equation implied by the given $C(12)$. Specifially, for the Percus-Yevick (PY) approximation used in this work, this is [1]

$g(12)=[1+S(12)] \exp [-\beta \phi(12)]$,

where $\phi(12)$ is the hard diatomics potential and $\beta=1 / k_{\mathrm{B}} T$. In this way, the discontinuities of $g(12)$ are exactly reproduced through the explicit Boltzmann factor of eq. (5).

In rough outline, the calculation to be carried out is then the following:

(a) Determine $C(12)$ from an approximation described below.

(b) Fourier transform to get

$$
\tilde{C}(12)=\int \mathrm{d} \boldsymbol{r}_{12} C(12) \exp \left(\mathrm{i} \boldsymbol{q} \cdot \boldsymbol{r}_{12}\right)
$$

(c) Compute the transform $\tilde{S}(12)$ from eq. (1). which now reads

$$
\tilde{S}(12)=\frac{\rho}{4 \pi} \int \mathrm{d} \omega_{3} \tilde{C}(13)[\tilde{C}(32)+\tilde{S}(32)] .
$$

(d) Invert to get

$$
S(12)=\frac{1}{(2 \pi)^{3}} \int \mathrm{d} \boldsymbol{q} \tilde{S}(12) \exp \left(-\mathrm{i} \boldsymbol{q} \cdot \boldsymbol{r}_{12}\right)
$$

(e) Determine $g$ (12) through eq. (5).

In practice, of course, the functional dependence of quantities such as $S(12))$ and $\tilde{S}(12)$ is far too detailed for their direct representation as stored arrays; instead, one has recourse to spherical harmonic expansions. With the $z$-axis along $r_{12}$. we have the simplest such representation [1]

$$
\begin{aligned}
S(12) & =S\left(r_{12}, \theta_{1}, \theta_{2}, \varphi_{12}\right) \\
& =4 \pi \sum_{l_{1} l_{2} m} S_{l_{1} l_{3} m}\left(r_{12}\right) Y_{l_{1} m}\left(\omega_{1}\right) Y_{l_{2} m}\left(\omega_{2}\right) .
\end{aligned}
$$

where $\bar{m}=-m$ and $\phi_{12}=\phi_{1}-\phi_{2}$ : similarly, in a rotated frame with $z$-axis along $\boldsymbol{q}$,

$$
\begin{aligned}
\tilde{S}(12) & =\tilde{S}\left(q, \theta_{1}^{\prime}, \theta_{2}^{\prime}, \phi_{12}^{\prime}\right) \\
& =4 \pi \sum_{l_{1} l_{2} m} \tilde{S}_{l_{1} l_{2} m}(q) Y_{l_{1} m}\left(\omega_{1}^{\prime}\right) Y_{l_{2} m}\left(\omega_{2}^{\prime}\right) .
\end{aligned}
$$

The calculation will now involve a finite set of spherical harmonic coefficients for each orientation-dependent function. Including indices up to 4 and taking account of the symmetries of the potential, these are, e.g., for $S(12)$, 


$$
\begin{array}{ccc}
S_{000} & S_{200} & S_{400} \\
& S_{220} & S_{420} \\
& & S_{440} \\
& S_{221} & S_{421} \\
& & S_{441} \\
& S_{222} & S_{422} \\
& & S_{442} \\
& & S_{443} \\
& & S_{444} .
\end{array}
$$

Because the spherical harmonics of eqs. (9) and (10) are referred to rotated axes, the connection between the sets $S_{l_{1} l_{2} m}(r)$ and $\tilde{S}_{l_{1} l_{2} m}(q)$ passes through Clebsch-Gordon transformation as well as Fourier. For full details on the derivation of this

Table 1

List of subroutines

$\begin{array}{ll}\text { DUMBELL } & \begin{array}{l}\text { Main program. Steps } 2.1,2.6,2.11 \text { and } 2.12 \text { are } \\ \text { performed in the main program, the remainder } \\ \text { in subroutines } \\ \text { Determine roots and Gaussian quadrature } \\ \text { weights of Legendre and Chebyshev polynomi- } \\ \text { als; calculate spherical harmonics at these roots }\end{array} \\ \text { SPACE } & \begin{array}{l}\text { CG transformation of spherical harmonic coeffi- } \\ \text { cients from axial set to space-fixed set (steps } 2.2\end{array} \\ \text { and 2.7) }\end{array}$

Table 2

Input data

\begin{tabular}{ll}
\hline NR & $\begin{array}{l}N_{r}, \text { number of points on } r \text { and } q \text { axes at which } \\
\text { arrays are calculated } \\
n, \text { number of points used in the Gaussian quadra- } \\
\text { tures }\end{array}$ \\
MDEG & $M$, largest value of index $m$ \\
RHOD3 & $\rho d^{3}$, reduced density \\
EL & $l$, interatomic separation in a molecule \\
SIGMA & $\begin{array}{l}\sigma, \text { diameter of an atom in a molecule } \\
\text { DR }\end{array}$ \\
\hline
\end{tabular}

and other steps of the calculation, we refer to refs. $[2,3]$, where additional references are also given. Here we proceed to discuss the implementation of these considerations in the program DUMBELL. See table 1 for the list of subroutines used and table 2 for the input data.

\section{Description of the program}

\subsection{Calculation of the DCF coefficients}

The analytic solution of the PY equation for hard spheres by Wertheim [4] and Thiele [5] has had far-reaching effects in the modern theory of simple fluids. The analogous solution for hard, homonuclear molecules is not yet known, but Pynn [6] has proposed an ad hoc generalization of the hard sphere result that works remarkably well in practice [7]. It is this empirical ansatz that is coded into DUMBELL, but it would be a straightforward matter to replace it with a true PY solution should one become available.

For the DCF, Pynn suggests

$$
\begin{aligned}
C(12) & =a+b\left[\frac{r_{12}}{\sigma(12)}\right]+c\left[\frac{\underline{r_{12}}}{\sigma(12)}\right]^{3}, r_{12}<\sigma(12), \\
& =0, \quad r_{12}>\sigma(12),
\end{aligned}
$$

where

$$
\begin{aligned}
& a=-(1+2 \eta)^{2} /(1-\eta)^{4}, \\
& b=6 \eta\left(1+\frac{1}{2} \eta\right)^{2} /(1-\eta)^{4}, \\
& c=\frac{1}{2} \eta a
\end{aligned}
$$

and

$$
\eta=\frac{1}{6} \pi \rho d^{3} .
$$

Here $\sigma(12)$ is the center-to-center separation between a pair of dumbell molecules at contact for a particular orientation of the pair and $\eta$ is the molecular packing fraction. The parameter

$d=\left(\sigma^{3}+\frac{3}{2} \sigma^{2} l-\frac{1}{2} l^{3}\right)^{1 / 3}$

is the diameter of a sphere having the same volume as the diatomic molecule, of which $\sigma$ is the atomic diameter and $l$ the interatomic separation. 
More explicitly, the dumbell potential is expressed in terms of the atom-atom separations. With the atoms of molecules 1 and 2 labelled respectively as $a, b$ and $a^{\prime}, b^{\prime}$. these distances are

$r_{\text {aa }}^{2}=A-B_{1}+B_{2}-C$.

$r_{\mathrm{ab}}^{2}=A-B_{1}-B_{2}+C$,

$r_{\mathrm{hi}}^{2}=A+B_{1}+B_{2}+C$.

$r_{\mathrm{hh}^{\prime}}^{2}=A+B_{1}-B_{2}-C$.

where

$A=r_{12}^{2}+\frac{1}{2} l^{2}$,

$B_{j}=r_{12} l \cos \theta_{j}$.

$C=\frac{1}{2} l^{2}\left(\cos \theta_{1} \cos \theta_{2}+\sin \theta_{1} \sin \theta_{2} \cos \phi_{12}\right)$.

The contact separation $\sigma(12)$ is determined by setting all $r_{\alpha \alpha^{\prime}}$ to $\sigma$ and solving eqs. (15) for the largest resulting $r_{12}$.

The coefficients $C_{l_{1} / m}(r)$ follow by inversion of the analogue of eq. (9),

$$
\begin{aligned}
C_{l_{1} / 2 m}\left(r_{12}\right)= & \frac{1}{4 \pi} \int \mathrm{d} \omega_{1} \mathrm{~d} \omega_{2} C(12) Y_{l_{1} m}^{*}\left(\omega_{1}\right) \\
& \times Y_{l_{2} \bar{m}}^{*}\left(\omega_{2}\right) .
\end{aligned}
$$

which is evaluated using n-point Gaussian quadratures as

$$
\begin{aligned}
C_{l_{1} / 2 m}(r)= & \frac{1}{n} \sum_{k_{1}, k_{2}, j=1}^{n} w_{k_{1}} w_{k_{2}} C\left(r, x_{k_{1}}, x_{k_{2}}, y_{1}\right) \\
& \times \mathscr{P}_{l_{1} m}\left(x_{k_{1}}\right) \mathscr{P}_{l_{2} m}\left(x_{k_{2}}\right)(-1)^{m} T_{m}\left(y_{j}\right) \\
\equiv & \left\langle C(12) \mid l_{1} l_{2} m\right\rangle .
\end{aligned}
$$

In eq. (18), the $x_{k}$ and $y_{j}$ are the $n$ zeroes of the Legendre polynomial $P_{n}(x)$ and the Chebyshev polynomial $T_{n}(y)$, respectively, the weights $w_{h}$ are

$w_{k}=\left\{\left(1-x_{k}^{2}\right)\left[P_{n}^{\prime}\left(x_{k}\right)\right]^{2}\right\}^{-1}$.

and $\mathscr{P}_{l m}(x)$ is the associated Legendre function normalized to 2 .

Depending on the largest value $M$ of the index $m$ specified in the input $(M=0,2$ or 4$)$, there will be respectively 1,5 or 14 coefficients $C_{h_{1} / 2 m}(r)$ calculated. (See the three columns of the $S(12)$ array displayed above.) For efficiency, a single index, rather than three, is used in the program to label these coefficients. The order in which they are arranged is given in the program commentary. as is that of the similarly labelled space-fixed arrays described below.

It is further convenient to keep these arrays multiplied by $r$ (and transforms by $q$ ), thereby avoiding the need to separately evaluate Fourier transforms for zero argument. This is indicated in the sequel of this paper by writing. e.g.. $\left[r C_{1, l_{2} m}(r)\right]$. with square brackets, for what in the program goes by the name associated with $C_{1_{1} / \ldots, \ldots}(r)$ alone.

Program input also requires the specification of $N_{r}$. number of, and $\Delta r$, interval between points on the $r$-axis, whereby all $r$-dependent functions are evaluated at the points

$r_{1}=j \Delta r, \quad j=1 \ldots, N_{r}-1$.

Because the Fast Fourier Transform (FFT) is used. $N$, must be an integer power of 2 .

\subsection{Transformation to space-fixed frame}

Preparatory to Fourier transformation, the DCF coefficients must be transformed to a space-fixed frame from the axial frame having $r$ along the $z$-axis. A Clebsch-Gordon (CG) transformation produces these new arrays as

$$
\begin{aligned}
{\left[r C\left(r: l_{1} l_{2} l\right)\right]=} & \sum_{m} \alpha_{m}\left\langle l_{1} m l_{2} \bar{m} \mid l_{1} l_{2} l 0\right\rangle \\
& \times\left[r C_{l_{1} l_{2} m}(r)\right] .
\end{aligned}
$$

where the angular brackets denote $C G$ coefficients, a factor $[(2 l+1) / 4 \pi]^{1 / 2}$ has been absorbed into the left-hand-side, and

$$
\begin{aligned}
\alpha_{m} & =1, & & m=0 \\
& =2, & & m>0 .
\end{aligned}
$$

The number of arrays in the space-fixed frame matches that in the axial frame.

\section{3. "Step-down" transformation}

The Fourier transform of a given $C\left(r: l_{1} l_{2} l\right)$ involves the spherical Bessel function $j_{l}(q r)$ of order $l$. For uniformity and simplicity, the coefficients with $l>0$ are first "lowered" to new func- 
tions $C^{(0)}\left(r ; l_{1} l_{2} l\right)$ by the recursive use of

$$
\begin{aligned}
& {\left[r C^{(k-2)}\left(r ; l_{1} l_{2} l\right)\right]=\left[r C^{(k)}\left(r ; l_{1} l_{2} l\right)\right]} \\
& -(2 k-1) r^{k} \int_{r}^{\infty} \mathrm{d} x \frac{\left[x C^{(k)}\left(x ; l_{1} l_{2} l\right)\right]}{x^{k}}
\end{aligned}
$$

starting from $\left[r C^{(l)}\left(r ; l_{1} l_{2} l\right)\right] \equiv\left[r C\left(r ; l_{1} l_{2} l\right)\right]$. With this transformation, all Fourier transforms can be computed using the single kernel $j_{0}(q r)=$ $\sin (q r) / q r$. The trapezoidal rule is used to evaluate the integral in (23).

\subsection{Fourier transformation}

The transforms

$\left[q \tilde{C}\left(q ; l_{1} l_{2} l\right)\right]=4 \pi \int_{0}^{\infty} \mathrm{d} r\left[r C^{(0)}\left(r ; l_{1} l_{2} l\right)\right] \sin q r$

are computed using the FFT. All transforms are evaluated at the points

$q_{j}=j \pi / N_{r} \Delta r, \quad j=1,2, \ldots, N_{r}-1$.

\subsection{Transformation to axial frame}

The next step requires generation of the coefficients of $\tilde{S}(12)$ from those of $\tilde{C}(12)$ through the $\mathrm{OZ}$ equation. This is more easily accomplished in an axial frame with $z$-axis along $q$. To this end, we perform another CG transformation (the inverse of $(21)$ ),

$$
\left[q \tilde{C}_{l_{1} l_{2} m}(q)\right]=\sum_{l}\left\langle l_{1} m l_{2} \bar{m} \mid l_{1} l_{2} l 0\right\rangle\left[q \tilde{C}\left(q ; l_{1} l_{2} l\right],\right.
$$

where we recall that the right-hand-side still contains the implicit factor introduced in eq. (21). Again, the number of coefficients remains unchanged.

\subsection{Solution of the $O Z$ equation}

With its angle-dependent functions expanded in their axial frame, the $\mathrm{OZ}$ equation (7) yields the solution

$$
\begin{aligned}
{\left[q \tilde{\mathbf{S}}_{m}(q)\right]=} & (-1)^{m} \rho\left\{q \mathbf{l}-(-1)^{m} \rho\left[q \tilde{\mathbf{C}}_{m}(q)\right]\right\}^{-1} \\
& \times\left[q \tilde{\mathbf{C}}_{m}(q)\right]^{2},
\end{aligned}
$$

where the matrices $\tilde{\mathbf{S}}_{m}(q), \tilde{\mathbf{C}}_{m}(q)$ have elements $\tilde{S}_{l_{1} l_{2} m}(q), \tilde{C}_{l_{1} l_{2} m}(q)$ with $l_{1}, l_{2}=m, m+2, \ldots, M$. When the coefficients of $\tilde{C}(12)$ become small enough with increasing $q$, the corresponding $\tilde{S}_{l_{1} l_{2} m}(q)$ are simply set to zero and eq. (27) bypassed.

\subsection{Transformation to space-fixed frame}

As before for $C(12)$, the coefficients of $\tilde{S}(12)$ must first be transformed to a space-fixed frame before the Fourier inversion can be carried out. This is effected by another CG transformation like that of eq. (21),

$$
\begin{aligned}
{\left[q \tilde{S}\left(q ; l_{1} l_{2} l\right)\right]=} & \sum_{m} \alpha_{m}\left\langle l_{1} m l_{2} \bar{m} \mid l_{1} l_{2} l 0\right\rangle \\
& \times\left[q \tilde{S}_{l_{1} l_{2} m}(q)\right] .
\end{aligned}
$$

\subsection{Inverse Fourier transformation}

The inverse of the transform of eq. (24) yields the "lowered" coefficients

$$
\begin{aligned}
{\left[r S^{(0)}\left(r ; l_{1} l_{2} l\right)\right]=} & \frac{1}{2 \pi^{2}} \int_{0}^{\infty} \mathrm{d} q\left[q \tilde{S}\left(q ; l_{1} l_{2} l\right)\right] \\
& \times \sin q r,
\end{aligned}
$$

which must next be put through a "raising" operation. As with (24), eq. (29) is evaluated using the FFT.

\section{9. "Step-up" transformation} is

The inverse of the lowering transformation (23)

$$
\begin{aligned}
& {\left[r S^{(k)}\left(r ; l_{1} l_{2} l\right)\right]=\left[r S^{(k-2)}\left(r ; l_{1} l_{2} l\right)\right]} \\
& \quad-\frac{(2 k-1)}{r^{k}} \int_{0}^{r} \mathrm{~d} x x^{k-1}\left[x S^{(k-2)}\left(x ; l_{1} l_{2} l\right)\right]
\end{aligned}
$$


applied recursively from the $S^{(1)}\left(r: l_{1} l_{2} l\right)$ until reaching $S\left(r ; l_{1} l_{2} l\right) \equiv S^{(l)}\left(r ; l_{1} l_{2} l\right)$. Because the integral in (30) is divided by $r^{h}$, the result could be particularly sensitive to quadrature errors at small $r$. To avoid these, a modified trapezoidal rule is used here, wherein only $x S(x)$ is fitted by linear interpolation.

\subsection{Transformation to axial frame}

A final CG transformation like that of (26).

$\left[r S_{l_{1}, l_{1}}(r)\right]=\sum_{l}\left\langle l_{1} m l_{2} \bar{m} \mid l_{1} l_{2} l 0\right\rangle\left[r S\left(r ; l_{1} l_{2} l\right)\right]$.

produces the axial coefficients from which $S(12)$, and so $g(12)$, can be conveniently reconstructed using eq. (9). For $S(12)$, this expansion converges rapidly, unlike that of the PDF itself [8].

\subsection{Calculation of the PDF coefficients}

Using the PY approximation (5), we obtain the axial coefficients of $g(12)$ as earlier in eq. (18) the DCF coefficients.

$g_{l_{1} l_{2} m}(r)=\left\langle[1+S(12)] \prod_{\alpha, \alpha r^{\prime}} \theta\left(r_{\alpha \alpha^{\prime}}-\sigma\right) \mid l_{1} l_{2} m\right\rangle$.

where now the Boltzmann factor of (5) has been written as a product of step functions which exclude contributions from orientations with overlapping molecules.

Finally, we recall that the DCF modelled by eq. (11) is qualitatively wrong for small $r$ [7]. This deficiency can be mitigated at this point by recalculating the DCF coefficients from

$C(12)=g(12)-1-S(12)$.

\subsection{Thermodynamics}

The thermodynamic quantities directly obtainable from the above are the virial pressure $p_{\mathrm{v}}$,

$$
\beta p_{v} / \rho=1+\frac{2}{3} \pi \rho\left\langle\sigma(12)^{3} g(\sigma(12)) \mid 000\right\rangle,
$$

and the compressibility $\chi$.

$$
\begin{aligned}
\left(\frac{\rho \chi}{\beta}\right)^{\prime} & =\beta\left(\frac{\partial p}{\partial \rho}\right)_{,} \\
& =1-4 \pi \rho\left\langle\int_{0}^{\infty} \mathrm{d} r r^{2} C(12) \mid 000\right\rangle .
\end{aligned}
$$

Using the PY relation $g=-C$ at contact and the modelled $C(12)$, it is easy to see that eq. (34) becomes [9]

$$
\begin{aligned}
\beta p_{v} / \rho & =1-\frac{2}{3} \pi \rho(a+b+c)\left\langle\sigma(12)^{3} \mid 000\right\rangle \\
& =1+\left(\beta p_{\mathrm{v}}^{(1 \mathrm{SS})} / \rho-1\right) B_{2}^{*},
\end{aligned}
$$

where $p_{s}^{(\mathrm{HS})}$ is the PY hard sphere virial pressure $[4,5]$ and

$$
B_{2}^{*}=\left\langle\sigma(12)^{3} \mid 000\right\rangle / d^{3}
$$

the (reduced) second virial coefficient. The latter has been analytically evaluated by Isihara [10]; in the program, however, it is calculated numerically.

Similarly, a change of variable from $r$ to $r / \sigma(12)$ reduces (35) to

$$
\begin{aligned}
\beta\left(\frac{\partial p}{\partial \rho}\right)_{T} & =1-4 \pi \rho\left(\frac{1}{3} a+\frac{1}{4} b+{ }_{6}^{\frac{1}{6}} c\right)\left\langle\sigma(12)^{3} \mid 000\right\rangle \\
& =1+\left[\beta\left(\frac{\partial p}{\partial \rho}\right)_{T}^{(\mathrm{HS})}-1\right] B_{2}^{*} .
\end{aligned}
$$

which can be integrated to yield the compressibility equation of state.

$\beta p_{\mathrm{c}} / \rho=1+\left(\beta p_{\mathrm{c}}^{(\mathrm{HS})} / \rho-1\right) B_{2}^{*}$,

in terms again of the hard sphere result [4.5] for the given $\eta$.

\section{Acknowledgement}

This work was supported by the National Science Foundation under Grant Number CHE8402144.

\section{References}

[1] W.B. Streett and K.E. Gubbins, Ann. Rev. Phys. Chem. 28 (1977) 373 .

[2] F. Lado, Mol. Phys. 47 (1982) 283. 
[3] F. Lado, Mol. Phys. 47 (1982) 299.

[4] M.S. Wertheim, Phys. Rev. Lett. 10 (1963) 321.

[5] E. Thiele, J. Chem. Phys. 39 (1963) 474.

[6] R. Pynn, Solid State Commun. 14 (1974) 29; J. Chem. Phys. 60 (1974) 4579.
[7] F. Lado, Mol. Phys. 54 (1985) 407.

[8] D.J. Tildesley, W.B. Streett and D.S. Wilson, Chem. Phys. 36 (1979) 63.

[9] E. Enciso, private communication.

[10] A.J. Isihara, J. Chem. Phys. 19 (1951) 397. 


\section{TEST RUN OUTPUT}
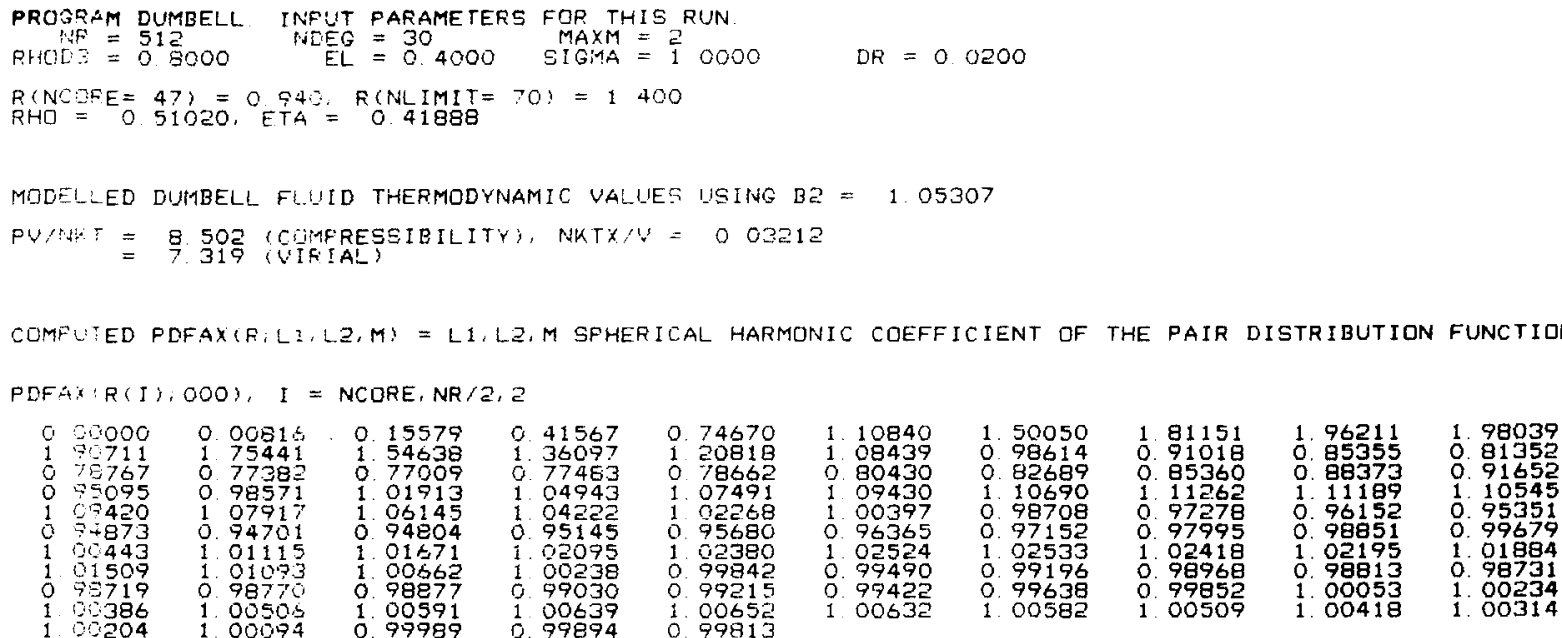

PDFAK(R I ; 200), I = NCORE, NR/2, 2
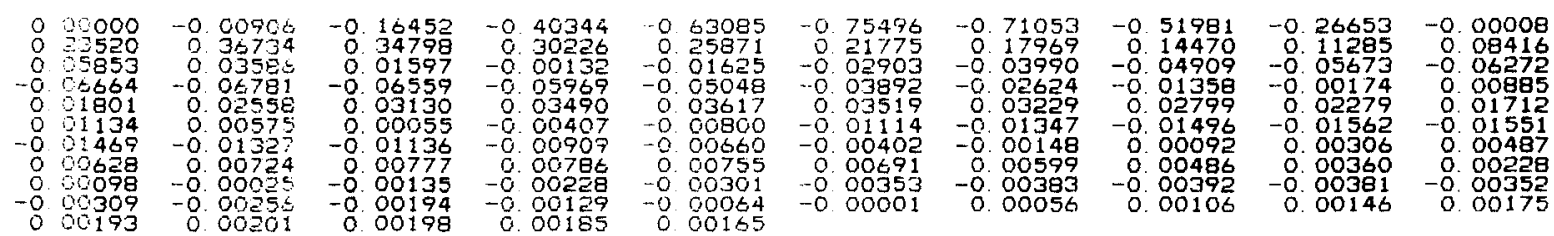

PDFAXIR(I); 220$), I=N C Q R E, N R / 2,2$
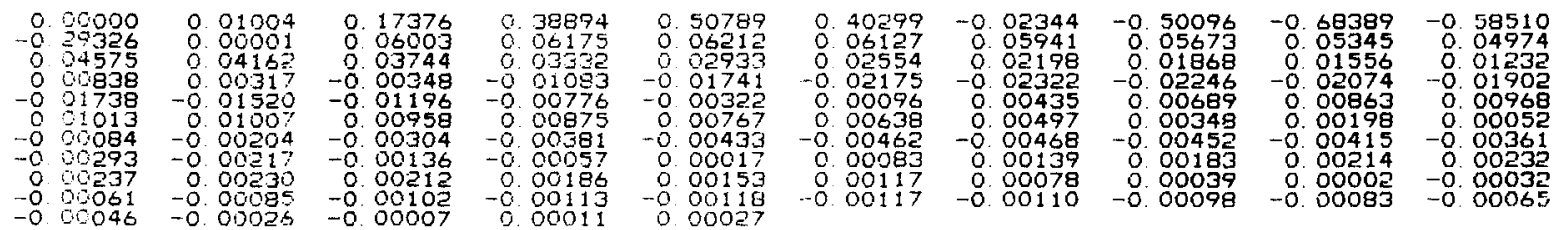
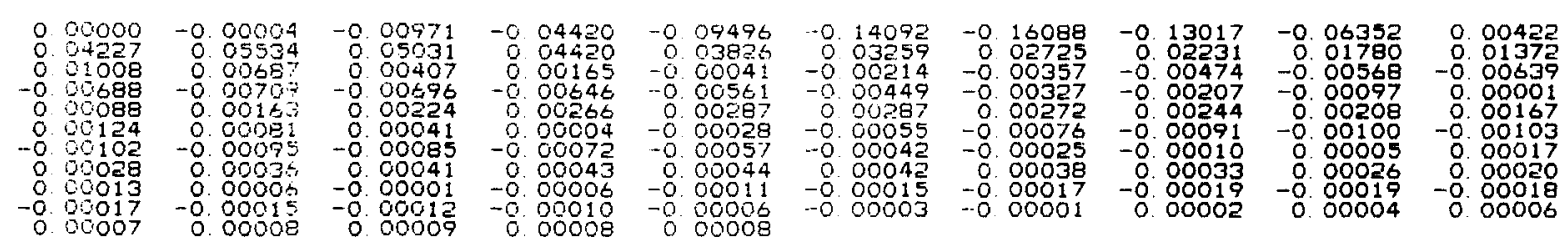

PDFAXIR(I)
0.90000
0.00996
-0.00070
-0.09042
0.00027
-0.00007
-0.90002
0.00003
-0.00001
0.0000
0.00000
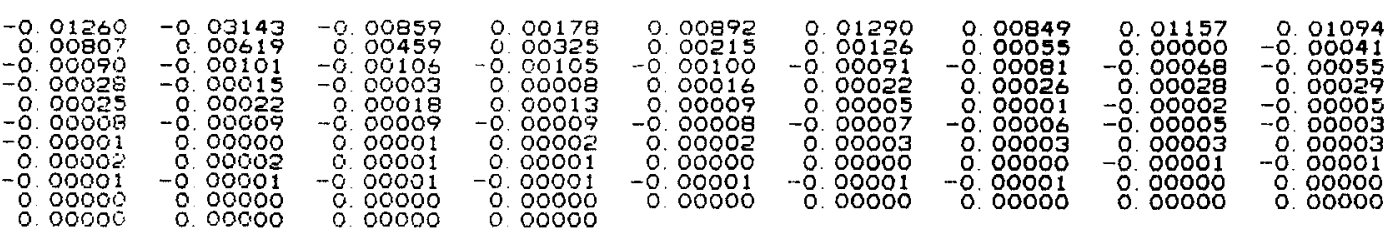